Platten (Schumann hart der Agfa) waren empfindlich, und Matta $\mathrm{ch} \mathrm{c}$. Ew ald haben entsprechend bei diesem empfindlichen Plattenmaterial nie einen Wendepunkt oder Schwellenwert in den Schwärzungskurven gefunden.

Zur Vereinfachung der Darstellung werden wir nicht $s_{2} / s_{1}$, sondern $\left(s_{2} / s_{1}-1\right) /\left(n_{2} / n_{1}-1\right)$ als Funktion von $s_{2}$ betrachten, was an der Gestalt der Kurve nichts ändert, da $n_{2} / n_{1}$ konstant ist.

Für Fall I hat man

a) für $n_{1} \approx n_{2}$ :

$$
\left(\frac{s_{2}}{s_{1}}-1\right) /\left(\frac{n_{2}}{n_{1}}-1\right) \approx \frac{n}{s} \frac{d s}{d n}=\frac{s-1}{s} \ln (1-s),
$$

b) für $n_{1} \ll n_{2}$ :

$$
\left(\frac{s_{2}}{s_{1}}-1\right) /\left(\frac{n_{2}}{n_{1}}-1\right) \approx \frac{s_{2}}{n_{2}}=-\frac{s_{2}}{\ln \left(1-s_{2}\right)} .
$$

Für Fall II ergibt sich

a) für $n_{1} \approx n_{2}$ :

$\left(\frac{s_{2}}{s_{1}}-1\right) /\left(\frac{n_{2}}{n_{1}}-1\right) \approx \frac{n^{2} \exp (-n)}{1-(1+n) \exp (-n)}$,

b) für $n_{1} \ll n_{2}$ :

$\left(\frac{s_{2}}{s_{1}}-1\right) /\left(\frac{n_{2}}{n_{1}}-1\right)=\infty$.

Abb. 7 zeigt für die Fälle Ia, Ib, II a und IIb die Funktion $\left(s_{2} / s_{1}-1\right) /\left(n_{2} / n_{1}-1\right)$ in Abhängigkeit von $s_{2}$. In Fall I wird für $s_{2}=0$ das Schwärzungsverhältnis mit dem Mischungsverhältnis identisch, in Fall II dagegen nicht. Verallgemeinernd kommen wir zu dem Ergebnis, daß das Verfahren der Extrapolation auf die Schwärzung Null nur anwendbar ist, wenn die Schwärzungskurve nicht ,durchhängt“, wenn sie also weder einen Wendepunkt noch einen Schwellenwert besitzt. Da bei den verwendeten S c h u man n - Platten diese Bedingung erfüllt war, konnte das besprochene Méßverfahren angewandt werden.

Weiter sehen wir aus Abb.7, daß in Fall I für alle Mischungsverhältnisse der Verlauf von $s_{2} / s_{1}$ zwischen $s_{2}=0$ und $s_{2}=0,5$ wenig gekrümmt ist, so daß

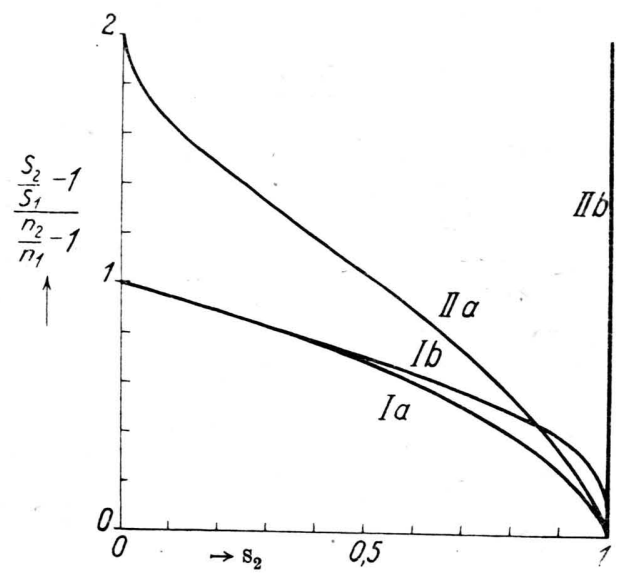

Abb. 7. Das Schwärzungsverhältnis $s_{2} / s_{1}$ bei konstantem Mischungsverhältnis $n_{2} / n_{1}$ als Funktion der Schwärzung $s_{2}$ bei Vorliegen der Schwärzungskurven $s=1-\exp (-n)$ (I) und $s=1-(1+n) \exp (-n)$

(II), wenn $n_{1} \approx n_{2}$ (a) und $n_{1} \ll n_{2}$ (b) ist.

man in diesem Gebiet mit einer geradlinigen Extrapolation auf die Schwärzung Null keinen großen Fehler begeht. (Man findet $n_{2} / n_{1}-1 \mathrm{um}$ höchstens $3 \%$ zu groß.)

Hrn. Prof. J. Mattauch, in dessen Abteilung ich die Untersuchung durchführen durfte, möchte ich für sein freundliches Interesse an der Arbeit und Frl. Scheld für ihre geschickten Dienste als Assistentin herzlich danken.

\title{
Ionisierungsspannung und Elektronenabschirmung im Periodischen System
}

\author{
Von Wolfgang Finkelnburg ${ }^{1}$
}

Herrn Prof. Dr. Hans Rau in Darmstadt zum 65. Geburtstag gewidmet

(Z. Naturforschg. 2 a, 16-20 [1947]; eingegangen am 9. Sept. 1946)

Die Abschirmung der auf das äußerste Elektron wirkenden Kernladung durch die inneren Elektronen zeigt im Periodischen System der Elemente Gesetzmäßigkeiten, die Schlüsse auf die Verhältnisse in der Elektronenhülle gestatten und die Extrapolation noch unbekannter sowie die Korrektur unsicherer Ionisierungsspannungen ermöglichen.

$\mathrm{D}^{\mathrm{i}}$ ie Ionisierungsenergie $E_{i}$ des im $n$-quantigen Zustand gebundenen Elektrons eines wasserstoffähnlichen Ions der Kernladung $Z e$ ist gleich dem Termwert des entsprechenden Energiezustands

\footnotetext{
1 z. Zt. Nördlingen/Bayern, Oskar-Mayer-Str. 10.
}

$$
E_{i}(n)=Z^{2} R / n^{2},
$$

wo $R$ die hier in $e$-Volt ausgedrückte $\mathrm{R} \mathrm{y} \mathrm{d} \mathrm{berg-}$ Konstante

$$
R=15,54 e-\text { Volt }
$$

ist. Bei einem beliebigen Atom der Ordnungszahl $N=Z$ wird ein Teil der Kernladung $Z$ in seiner 
Wirkung auf das äußerste Elektron durch die $(Z-1)$ inneren Elektronen abgeschirmt. Da das abzutrennende äußerste Elektron sich dann im Feld der effektiven Kernladung $Z_{\text {eff }}<Z$ befindet, gilt für die Ionisierungsenergie $E_{i}$

$$
E_{i}=Z_{\text {eff }}^{2} R / n^{2},
$$

wo jetzt $n$ die Hauptquantenzahl des äußersten Elektrons, also z.B. 2 für Lithium und 7 für Radium ist.

Bei empirisch bekannter Ionisierungsspannung $E_{i}$ und aus dem Periodischen System bekanntem $n$ kann man also wegen (2) die effektive Kernladung

$$
Z_{\text {eff }}=n \sqrt{E_{i} / R}
$$

berechnen. Die Abschirmungszahl

$$
s=Z-Z_{\mathrm{eff}}
$$

gibt dann die gesamte Abschirmung durch die inneren Elektronen an. $\mathrm{Zu}$ interessanten Gesetzmäßigkeiten gelangt man nun, wenn man die $\mathrm{Ab}$ schirmungszunahme

$$
\Delta s=s_{N}-s_{N_{-1}}
$$

( $N=$ Ordnungszahl) beim Fortschreiten von einem Element zum nächsten im Periodischen System betrachtet. Diese $\Delta s$-Werte wurden nach (4), (5) und (6) für alle Atome und Atomionen berechnet, für die spektroskopische oder experimentelle Werte der Ionisierungsspannung $E_{i}$ bekannt sind. Für die neutralen Atome sind in Tab. 1 die Ionisierungsspannungen und die Werte der Abschirmungszunahme $\Delta s$ gegenüber dem jeweils vorhergehenden Element angegeben.

Man erkennt, daß für diese letzte Größe auffallende Gesetzmäßigkeiten bestehen. So ist z. B. die Abschirmungszunahme $\Delta s$ bei den Halogenen und der unter sich besonders ähnlichen Gruppe $\mathrm{Zn-Cd-Hg}$ annähernd bzw. genau konstant gleich 0,68; sie ist bei den Erdalkalien, der KohlenstoffGruppe und den Edelgasen ebenfalls klein und nimmt mit zunehmender Ordnungszahl in der Gruppe leicht ab. $\Delta s$ ist dagegen recht groß $(>1)$ und wächst erheblich mit zunehmender Ordnungszahl bei den Alkalien und Erden. Aber auch die feineren Einzelheiten der $\Delta s$-Werte in Tab. 1 sind nicht die Wirkung zufälliger Schwankungen. So kehrt die Folge der $4 s$-Werte der Edelgas-Gruppe mit ihrem auffallenden Maximum beim zweiten Glied (Ne) nach Tab. 2 sehr genau wieder in den Gruppen der edelgasähnlichen Ionen .

Ähnlich ist es mit dem $\Delta s$-Verlauf in den übrigen Gruppen des Periodischen Systems. Der Zusammenhang dieser durch die Heranziehung der Ionen vielfach gesicherten Gesetzmäßigkeiten mit den Quantenzahlen der zuletzt eingebauten Elektronen ist offenbar: Die Abschirmungszunahme ist groß und nimmt mit wachsender Ordnungszahl in den Gruppen erheblich zu beim Einbau des ersten $s$ Elektrons und des ersten $p$-Elektrons jeder Elektronenschale; sie ist umgekehrt klein beim weiteren Einbau gleichartiger Elektronen, d. h. beim zweiten $s$-Elektron (2. Gruppe) und dem 2., 3., 5. und 6. p-Elektron (4., 5., 7. und 8. Gruppe).

Die aus Tab. $1 \mathrm{zu}$ entnehmenden empirischen Gesetzmäßigkeiten sind zum großen Teil qualitativ theoretisch verständlich, einige von ihnen aber auch sehr überraschend und für ein anschauliches Verständnis des Aufbaues der Elektronenhüllen von Interesse. Daß die Abschirmung beim Einbau des ersten $s$-Elektrons jeder Elektronenschale um mehr als eine Einheit zunimmt, erscheint verständlich, da vom Standpunkt des weit außen angebauten Elektrons aus sämtliche Elektronen des Rumpfes wesentlich stärker abschirmen als vom Standpunkt des vorher eingebauten letzten $p$-Elektrons der vorhergehemden Schale aus. Die Abschirmungszunahme beim Anbau des ersten $s$ Elektrons muß daher in Übereinstimmung mit Tab. 1 um so größer sein, je mehr Elektronen bereits in tieferen Schalen sitzen, d. h. je größer die Ordnungszahl des Alkaliatoms ist. Diese $\mathrm{Zu}$ nahme von $\Delta s$ mit $N$ wird noch verstärkt dadurch, daß nach der Boh r schen Theorie der Bahnradius mit dem Quadrat der Hauptquantenzahl (= Schalennummer) $n$ zunimmt, das erste $s$-Elektron mit zunehmendem $n$ also in relativ immer größerem Abstand vom Rumpf eingebaut wird. In ähnlicher Weise ist die Größe und Zunahme der $\Delta s$-Werte beim Einbau des ersten $p$-Elektrons jeder Schale bei den Erden $\mathrm{B}, \mathrm{Al}$, Ga, In, Tl zu erklären, ó̄wohl hier die Größe der $\Delta s$-Werte etwas überrascht, da der mittlere Kernabstand eines $p$-Elektrons ja nicht viel größer ist als der der $s$-Elektronen der gleichen Schale.

\footnotetext{
2 Dieser Gang ist theoretisch daraus verständlich. daß beim He ein zweites $s$-Elektron eingebaut wird, es in dieser Hinsicht also über dem Be stehen müßte, während bei den übrigen Edelgasen das letzte $p$ Elektron eingebaut wird.
} 


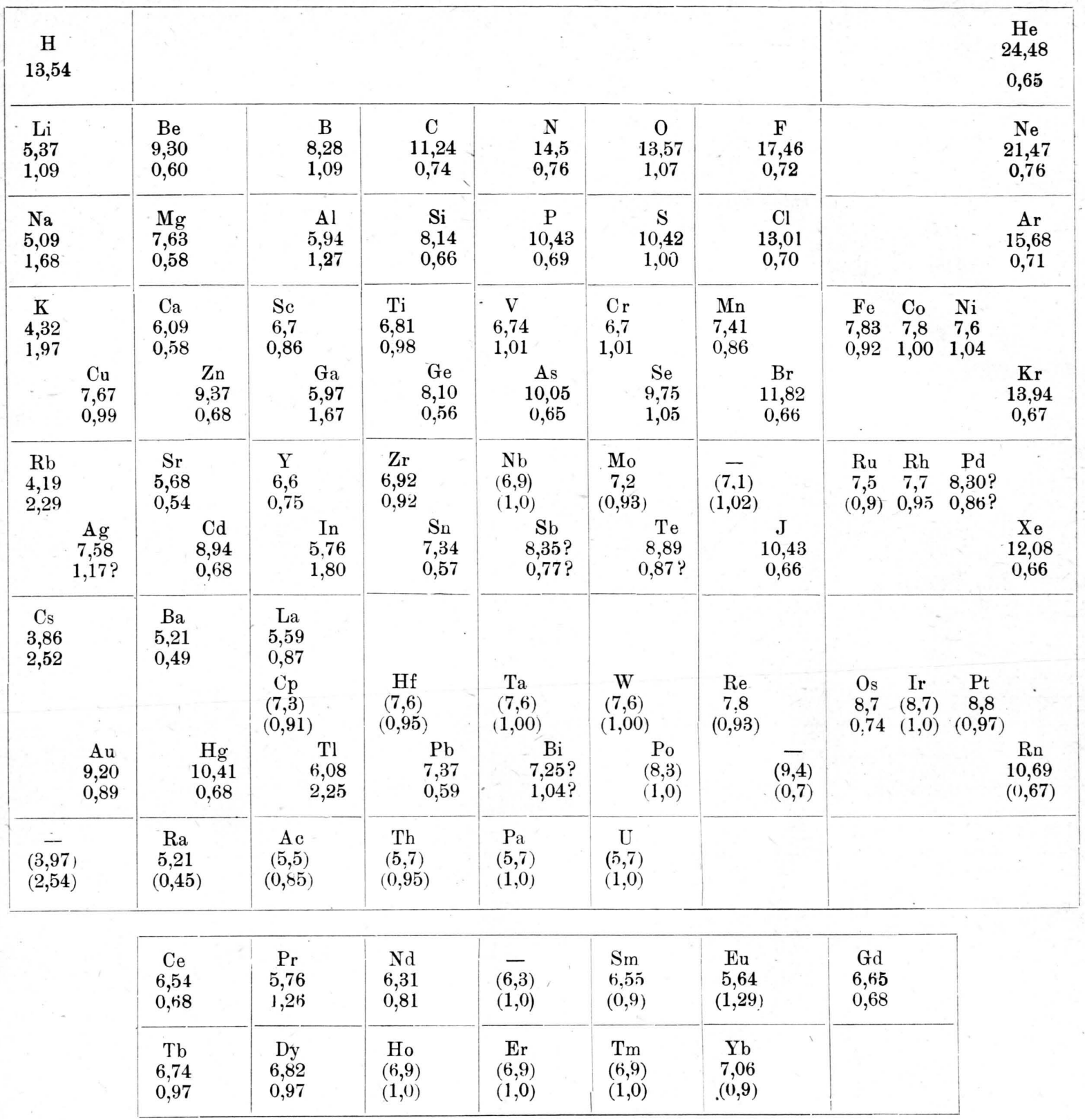

Tab. 1. Verteilung der Ionisierungsspannungen $E_{i}$ und der Abschirmungsänderungen $\Delta s$ über das Periodische System. Eingeklammerte Werte vom Verf. berechnet; Werte mit Fragezeichen im Text korrigiert.

\begin{tabular}{|c|c|c|c|c|}
\hline$\Delta \mathrm{s}$ & $\Delta \mathrm{s}$ & $\Delta \mathrm{s}$ & $\Delta \mathrm{s}$ & $\Delta \mathrm{s}$ \\
\hline $\mathrm{He} 0,65$ & $\mathrm{Li}^{+} 0,64$ & $\mathrm{Be}^{++} 0,64$ & $\mathrm{~B}^{+++} 0,63$ & $\mathrm{C}^{++++} 0,63$ \\
$\mathrm{Ne} 0,76$ & $\mathrm{Na}^{+} 0,76$ & $\mathrm{Mg}^{++} 0,75$ & $\mathrm{Al}^{+++} 0,75$ & $\mathrm{Si}^{++++} 0,73$ \\
$\mathrm{Ar} 0,71$ & $\mathrm{~K}^{+} 0,71$ & $\mathrm{Ca}^{++} 0,70$ & $\mathrm{Ga}^{+++} 0,69$ & $\mathrm{Ge}^{++++} 0,69$ \\
$\mathrm{Kr} 0,67$ & $\mathrm{Rb}^{+} 0,70$ & $\mathrm{Sr}^{++} 0,69$ & $\mathrm{In}^{+++} 0,67$ & $\mathrm{Sn}^{++++} 0,66$ \\
$\mathrm{Xe} 0,66$ & $\mathrm{Cs}^{+} 0,68$ & $\mathrm{Ba}^{++} 0,67$ & $\mathrm{Tl}^{+++} 0,66$ & $\mathrm{~Pb}$ \\
\hline
\end{tabular}

Tab. 2. Werte der Abschirmungszunahme $\Delta s$ bei den Edelgasen und den edelgasähnlichen Ionen. 


\begin{tabular}{|l||c|c|c|c|c|c|c|c|c|}
\hline $\mathrm{N}$ & 41 & 43 & 61 & 67 & 68 & 69 & 71 & 72 & 73 \\
$\mathrm{El}$. & $\mathrm{Nb}$ & - & - & $\mathrm{Ho}$ & $\mathrm{Er}$ & $\mathrm{Tm}$ & $\mathrm{Cp}$ & $\mathrm{Hf}$ & $\mathrm{Ta}$ \\
$\mathrm{E}_{i}$ & 6,9 & 7,1 & 6,3 & 6,9 & 6,9 & 6,9 & 7,3 & 7,3 & 7,6 \\
$\mathrm{~N}$ & $\mathbf{7 4}$ & 84 & 85 & 87 & 89 & 90 & 91 & 92 & \\
$\mathrm{El}_{1}$ & $\mathrm{~W}$ & $\mathrm{Po}$ & - & - & $\mathrm{Ac}$ & $\mathrm{Th}$ & $\mathrm{Pa}$ & $\mathrm{U}$ & \\
$\mathrm{E}_{i}$ & 7,6 & 8,3 & 9,4 & $\mathbf{4 , 0}$ & $\mathbf{5 , 5}$ & $\mathbf{5 , 7}$ & $\mathbf{5 , 7}$ & $\mathbf{5 , 7}$ & \\
\hline
\end{tabular}

Tab. 3. Durch Interpolation der $\Delta s$-Werte ermittelte, bisher unbekannte Ionisierungsspannungen neutraler Atome (in $e$-Volt).

Daß beim Einbau eines zweiten oder dritten gleichartigen Elektrons die Abschirmung sich relativ wenig $(0,6$ bis 0,7$)$ ändert, scheint wieder anschaulich klar. Daß dagegen der Einbau des ersten $p$-Elektrons mit entgegengesetzter Spinrichtung wie der vorhergehenden in der 6. Gruppe $(\mathrm{O}, \mathrm{Se}, \mathrm{S})$ eine Anderung der Abschirmung um eine volle Einheit bewirkt, ist nicht ohne weiteres verständlich und deutet auf eine anschaulich noch nicht erklärte Wirkung des Spins hin. Dieses Ergebnis ist aber nicht nur durch die wenigen Zahlen der Tab. 1 gesichert, sondern zusätzlich durch die Abschirmungszahlen der ein- bis siebenfach ionisierten Atome, wobei unsere Regel sich ausnahmslos bestätigte.

Die relativ große durchschnittliche Abschirmungszunahme von 0,95 beim Einbau der inneren $d$-Elektronen bei den Elementen 21 bis 29 und 39 bis 45 entspricht durchaus der Erwartung, da die Ionisierung ja das äußerste $s$-Elektron betrifft, dem gegenüber der Einbau eines inneren $d$-Elektrons die Abschirmung um ungefähr eine Einheit vergrößern muß. Zu einem interessanten Ergebnis kommen wir, wenn wir aus den Ionisierungsspannungen der zweifach und höher ionisierten Atome die Abschirmungszahlen beim schrittweisen $A n$ bau der zehn $d$-Elektronen ohne überlagerte äußere $s$-Elektronen berechnen. Beim Anbau des zweiten bis fünften und des siebten bis zehnten $3 d$-Elektrons finden wir ein $\Delta s$ von 0,85 , beim Anbau des sechsten $3 d$-Elektrons dagegen ein $\Delta s$ von 1,15. Beim Anbau der zehn 4d-Elektronen liegen beide Werte um rund 0,1 niedriger. Wie bei den p-Elektronen erfolgt also auch beim ungestörten Anbau des ersten $d$-Elektrons mit entgegengesetzter Spinrichtung wie der fünf vorhergehenden ein Abschirmungssprung.

Bei den neutralen Atomen der Seltenen Erden entspricht die mittlere Abschirmungszunahme von 0,96 wieder der Erwartung, da der Einbau der inneren f-Elektronen die Abschirmung gegenüber dem abzutrennenden äußersten $s$-Elektron wieder um ungefähr eine Einheit vergrößern muß. Die großen Schwankungen der Einzelwerte von 0,68 beim Ce und Gd bis fast 1,3 beim Pr und Eu sind aber mit der Annahme, daß stets ein äußerstes $s$-Elektron bei der Ionisierung abgetrennt und ein inneres $f$-Elektron eingebaut wird, nicht vereinbar. Entweder liegen also bei den Seltenen Erden noch Fehler in den experimentellen Ionisierungsspannungen vor (was bei der benutzten Methode nicht unmöglich scheint), oder der Elektroneneinbau erfolgt bei diesen Atomen nicht regelmäßig, sondern unter gelegentlichem Hereinziehen und nachträglichem Wiederanbau äußerster Elektronen. Für die Aufklärung der Feinheiten des Elektronenschalenbaues wie für das Verständnis der Wirkung des Elektronenspins auf die Abschirmung können die hier besprochenen Gesetzmäßigkeiten also von Wert sein.

Unabhängig von diesen teilweise noch offenen Fragen der Deutung der $\mathcal{A}$-Gesetzmäßigkeiten bieten diese aber die Möglichkeit, die ungefähre Größe von $4 s$ und mittels (3) bis (6) damit die Ionisierungsspannungen solcher Atome $\mathrm{zu}$ berechnen, für die diese noch nicht bekannt sind. Die so ermittelten Werte von $\Delta s$ und $E$, sind in Tab. 1 eingeklammert. Tab. 3 gibt eine Úbersicht über die so neu bestimmten Ionisierungsspannungen neutraler Atome, wobei bei den Seltenen Erden ein regelmäßiger Einbau von $f$-Elektronen vorausgesetzt ist.

Auch eine Korrektur unsicherer $\boldsymbol{E}_{i}$-Werte ist grundsätzlich mittels unserer Gesetzmäßigkeiten möglich. Vergleicht man z. B. die $\Delta s$-Werte von $\mathrm{Sb}$ und Te mit den darüber stehenden je drei Elementen, so erscheint der $\Delta s$-Wert des Sb um etwa 0,13 zu groß, der des Te um den gleichen Betrag zu klein. Diese Korrektur würde (ohne Veränderung der Ionisierungsspannung des Te) eine Erhöhung der nach den Literaturangaben noch unsicheren Ionisierungsspannung des $\mathrm{Sb}$ von 8,35 auf 8,9 Volt ergeben. Für die nach der Literatur ebenfalls noch etwas zweifelhafte Ionisierungsspannung des Bi möchten wir aus den gleichen Gründen sogar eine Erhöhung von 7,25 auf 8,3 Volt für richtig halten. Beide Korrekturen gewinnen dadurch erheblich an Wahrscheinlichkeit, daß die Berechnung der $\Delta s$-Werte für einfach bis fünffach ionisierte Atome durchweg für den Einbau des dritten $p$-Elektrons (wie beim $\mathrm{Sb}$ und $\mathrm{Bi}$ ) 
eine Abschirmungszunahme von nur 0,65, beim Einbau des vierten $p$-Elektrons dagegen eine solche von 1,0 ergab.

Noch nicht sicher entschieden werden kann dagegen, ob die auffallend große Schwankung der $\Delta s$-Werte in der Reihe $\mathrm{Cu}-\mathrm{Ag}$-Au reell ist und auf der Sonderstellung des nur einwertig auftretenden Ag beruht, da eine Erniedrigung der Ionisierungsspannung des dem $\mathrm{Ag}$ vorhergehenden $\mathrm{Pd}$ von dem nicht sehr sicheren Wert von 8,3 auf 7,7 Volt den $\Delta s$-Wert des Pd dem des über ihm stehenden $\mathrm{Ni}$, und den des $\mathrm{Ag}$ dem des $\mathrm{Cu}$ anpassen würde.
Da andererseits der Unterschied der Elektronenanordnungen des Grundzustandes von Ni und $\mathrm{Pd}$ einerseits ${ }^{3}$ und der im atomphysikalisch-chemischen Verhalten des $\mathrm{Ag}$ gegenüber $\mathrm{Cu}$ und $\mathrm{Au}$ andererseits reelle $\Delta s$-Unterschiede zwischen diesen übereinander stehenden Atomen durchaus wahrscheinlich macht, muß die Ag-Pd-Frage noch offen bleiben, bis der Zusammenhang der $\Delta s$ Werte mit der Elektronenanordnung theoretisch genauer geklärt ist.

3 Vergl. z. B. A. So m me r feld, „Atombau und Spektrallinien“", 6. Aufl. Bd. I, S. 496.

\title{
Lichtabsorption und Molekularzustand des Jods in Dampf und Lösung
}

\author{
Von Gustav Kortüm und Gerd Friedheim \\ Aus dem Physikalisch-Chemischen Institut der Universität Tübingen \\ (Z. Naturforschg. 2 a, 20-27 [1947]; eingegangen am 21. Sept. 1946)
}

\begin{abstract}
Frühere Messungen sowohl wie die zu ihrer Deutung herangezogenen Hypothesen über den Molekularzustand des Jods in seinen violetten und seinen braunen Lösungen erweisen sich als unzureichend und widerspruchsvoll. Quantitative spektrographische Aufnahmen des Absorptionsspektrums von dampfförmigem Jod bei verschiedenen Temperaturen und Drucken sowie von Jodlösungen in Cyclohexan und Äther ergeben eine vollkommene Analogie der Spektren, deren Banden den gleichen Elektronenübergängen zugeordnet werden müssen. Das B e e r sche Gesetz ist im Bereich aller Banden gültig, lediglich eine schwache, im nahen UV gelegene Bande, die auch im Gaszustand kontinuierlich ist, zeigt starke Druck- bzw. Konzentrationsabhängigkeit, die auf ein Assoziationsgleichgewicht des Jods sowohl in gasförmiger wie in flüssiger Phase hinweist. Kryoskopische Messungen in Cyclohexan und Dioxan bestätigen dieses Ergebnis. Der charakteristische Farbunterschied der braunen und der violetten Jodlösungen kann danach nicht auf einen verschiedenen Dispersitätsgrad des gelösten Jods zurückgeführt werden. Er beruht wahrscheinlich auf der Wirkung verschiedenartiger zwischenmolekularer Kräfte und verschiedener Raumerfüllung, ohne daß man die Entstehung stöchiometrischer Additionsverbindungen annehmen muß.
\end{abstract}

$\mathrm{D}$ er Molekularzustand des Jods in seinen Lösungen ist bis in die neueste Zeit hinein der Gegenstand ungewöhnlich zahlreicher Untersuchungen gewesen. Hervorgerufen wurde dieses Interesse durch den charakteristischen Farbunterschied der ,,violetten“ und der „braunen“Jodlösungen, der als Kennzeichen eines verschiedenen Molekularzustandes des Jods in den beiden Gruppen von Lösungsmitteln aufgefaßt wurde. Zur Lösung dieses Problems. wurden außer optischen Messungen auch zahlreiche andere Methoden herangezogen, u. a. Bestimmungen des Verteilungsgleichgewichts zwischen verschiedenen Lösungsmitteln, kryoskopische Messungen, Diffusionsmessungen, Dampfdruckmessungen, magnetische Mes- sungen. Die Ergebnisse dieser Untersuchungen waren, ebenfalls bis in die neueste Zeit hinein, so widerspruchsvoll, daß das Problem noch immer als ungelöst betrachtet werden muß, wie schon daraus hervorgeht, daß immer neue Hypothesen zur Deutung der Versuche aufgestellt wurden. Wir haben deshalb im Lauf unserer Messungen über die Spektren der Halogene in Abhängigkeit vom Zusatz nicht absorbierender Fremdgase ( sog. Fremdgaseffekt) auch die Lichtabsorption des Jods im Gaszustand und in seinen Lösungen untersucht. Die Messungen führen zu eindeutigen Schlüssen über den Molekularzustand des Jods in gasförmiger und kondensierter Phase, worüber im folgenden berichtet wird. Eine kurze Über- 